\title{
Multifocal mucosa-associated lymphoid tissue lymphoma involving the lungs and the stomach: a rare clinical entity: a case report
}

\author{
Carla PM Lam ${ }^{1}$ *, MB, BS, CF Wong ${ }^{2}$, FHKCP, FRCP (Edin)
}

${ }^{1}$ Department of Medicine, Queen Mary Hospital, Pokfulam, Hong Kong

${ }^{2}$ Tuberculosis and Chest Medicine Unit, Grantham Hospital, Wong Chuk Hang, Hong Kong

* Corresponding author: meicarlalam@gmail.com

Hong Kong Med J 2019;25:243-5

https://doi.org/10.12809/hkmj176973

\section{Case report}

A 77-year-old male ex-smoker initially presented to Queen Mary Hospital, Hong Kong in April 2009 with an abnormal lung lesion at the left lower lobe on computed tomography (CT) scan of the abdomen during workup for abdominal aorta aneurysm (Fig 1). The lung lesion persisted despite antibiotic treatment. Flexible bronchoscopy, bronchial brushings and aspirate were unremarkable. It was decided to keep the patient under observation.

One year later he developed per-rectal bleeding. Colonoscopy was unremarkable but upper endoscopy revealed a $5-\mathrm{cm}$ ulcerative growth in the posterior wall of the proximal gastric body. Histology showed sheets of small abnormal lymphoid cells with pale cytoplasm and cleaved nuclear outline, strongly positive for CD20 and CD79a, and negative for CD3, CD5, CD23, CD10, CD43, and Cyclin-D1. There was lambda light chain restriction. The Ki-67 proliferation index was less than $10 \%$. Lymphoepithelial lesions were identified. Helicobacter pylori was not detected and the patient was treated as a case of isolated primary $\mathrm{H}$ pylorinegative gastric mucosa-associated lymphoid tissue (MALT) lymphoma with $H$ pylori eradication followed by rituximab for eight cycles. The response was suboptimal and he was subsequently prescribed three cycles of rituximab, cyclophosphamide, vincristine, and prednisolone.

He was admitted to our chest unit in July 2017 for treatment of chest infection. Review of serial chest X-ray and CT scans showed progressive deterioration of the lung lesion that had extended to the whole left lower lobe with consolidative changes, air bronchogram, multiple cystic changes and bronchial dilatation (Fig 2). Serial positron emission tomography-CT scans showed that the lung lesion was metabolically active with partial improvement upon chemotherapy correlating with that of the gastric MALT lymphoma. Repeat fibre-optic bronchoscopy and transbronchial lung biopsy revealed respiratory mucosa with diffuse dense lymphoid proliferation in the stroma. Lymphoepithelial lesions were again observed, positive for CD20 and negative for CD3,

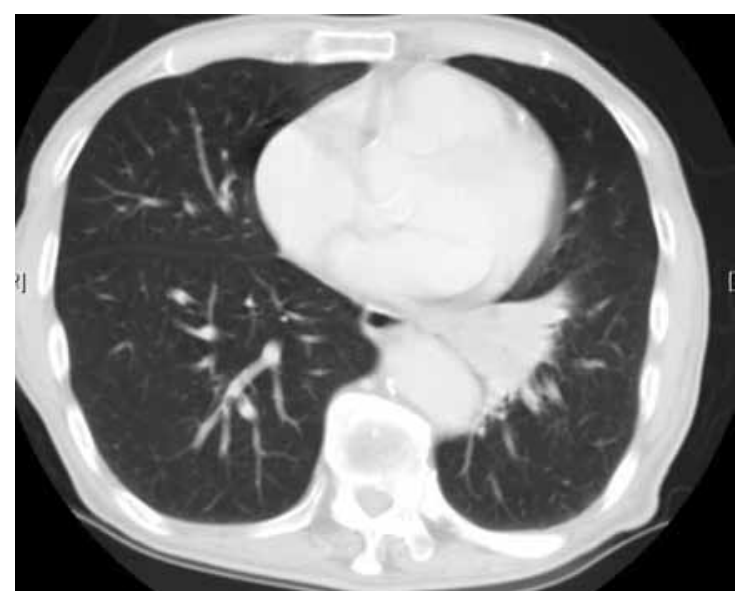

FIG I. Computer tomography of the thorax of a 77-yearold man on initial presentation showing left lower lobar consolidation

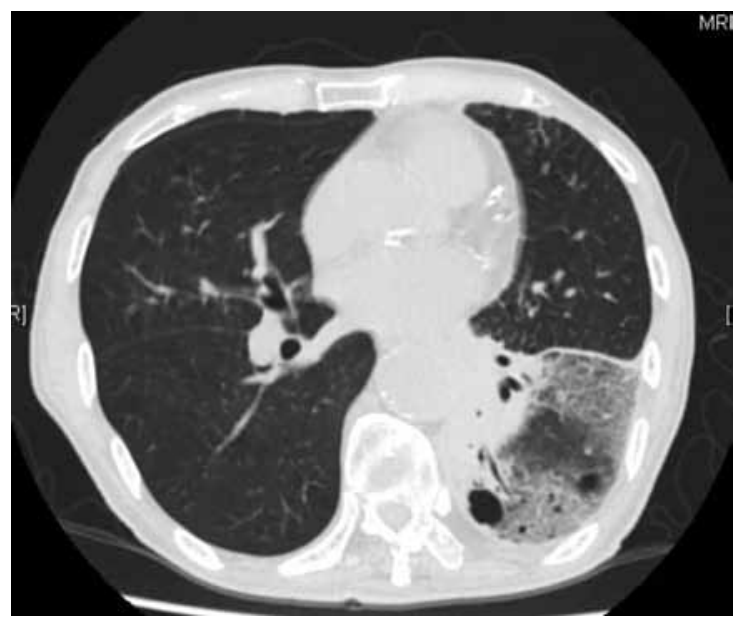

FIG 2. Reassessment computer tomography of the thorax of a 77-year-old man showing bilateral multiple cystic lung lesions, bronchial dilatation, and air-bronchogram

CD5, CD10, CD23, and Cyclin-D1. Lambda light chain restriction was demonstrated, compatible with MALT lymphoma. 


\section{Discussion}

Mucosa-associated lymphoid tissue lymphoma is a relatively rare disease with an annual incidence estimated at 1/313000; MALT lymphoma accounts for $6 \%$ to $8 \%$ of all non-Hodgkin lymphomas. Histologically, MALT lymphoma is characterised by neoplastic cell infiltration around reactive secondary lymphoid follicles in a marginal zone distribution and centrocyte-like cells that are small-to-medium in size with small irregular nuclei. Neoplastic cells frequently have abundant pale cytoplasm and a distinct cell border, resembling small mature lymphocytes. Lymphoepithelial lesions have been frequently described. The immunophenotype of MALT lymphoma is virtually identical to that of non-neoplastic marginal-zone $B$ cells. They are positive for CD20 but negative for IgD, CD5, CD10, Bcl6, and Cyclin-D1. Demonstration of immunoglobulin light chain restriction is also helpful to exclude reactive lymphoid infiltrate. No specific immunohistochemical marker has been identified for MALT lymphoma with different tissues of origin. ${ }^{1}$

The most common site of involvement of MALT lymphoma is the stomach, accounting for half of all cases. Other sites include the small intestine (20\%-30\%), colon (10\%), salivary glands, thyroid, lung, bladder, and skin. Gastric MALT lymphoma is strongly associated with $H$ pylori that has been implicated in its pathogenesis. The majority (92\%98.3\%) of gastric MALT lymphomas are positive for $H$ pylori, and $H$ pylori eradication alone achieves complete remission of gastric MALT lymphoma in $80 \%$ of cases.

Pulmonary MALT lymphoma is a very rare condition, accounting for aproximately $1 \%$ of cases. Pulmonary MALT lymphoma is usually an indolent disease and has no association with $H$ pylori but is associated with chronic inflammatory conditions instead. Radiological features of pulmonary MALT lymphoma are diverse and include air bronchogram, bronchial dilation, nodular lesions, lung mass, ground-glass opacities, and cystic lung lesions. Lesions are likely multiple, bilateral without lobar predilection with maximum standardised uptake value varying from 2.8 to $9.4 .^{2}$

Although MALT lymphoma was once thought to be an indolent disease due to its tendency to remain localised for a prolonged period to the tissue of origin, multifocal involvement of MALT lymphoma at presentation has been increasingly reported in recent years. ${ }^{3,4}$ In a case series of 304 patients with MALT lymphoma in Japan, ${ }^{3}$ seven (2\%) had multifocal involvement, mostly involving the gastrointestinal tract. In another case series in Austria involving 72 patients with non-gastric MALT lymphoma, 23 (32\%) had multifocal disease either on presentation or during the study period.
Site-specific involvement was reported. The stomach was involved at staging upper endoscopy and histologically confirmed $(\mathrm{P}<0.0001)$ in seven of 13 patients with primary lung MALT lymphoma. ${ }^{4}$

There are no specific histological or immunostaining characteristics for MALT lymphoma at different sites. Sequence analysis of immunoglobulin heavy chain gene $(\operatorname{IgH})$ may help demonstrate multifocality of MALT lymphoma. In a study that recruited 170 patients with MALT lymphoma over 8 years, 11 had multifocal involvement and paired tumour biopsy samples were analysed in four. ${ }^{5}$ Monoclonal rearrangement of the IgH gene was detected in all four tumour pairs of which three had different VDJ sequences, indicating that there was no clonal relationship between the tumour pairs whereas the fourth demonstrated clonal identity. That study implied that MALT lymphomas involving different organ systems more often represent different clones and arise independently instead of disseminating from one system to another. ${ }^{5}$

In ourcase, we believe that the MALT lymphoma was multifocal in origin and the pulmonary lesion preceded that of the stomach based on the temporal sequence (appearance of lung lesion long before clinical manifestation of gastric MALT lymphoma). Further analysis of the VDJ sequence of the $\mathrm{IgH}$ of the tumour samples is needed to demonstrate the clonal relationship between them.

\section{Conclusion}

Mucosa-associated lymphoid tissue lymphoma was once thought to be an indolent disease localised to one tissue origin, but occurrence of multifocal disease has been increasingly reported. Our case illustrates multifocal MALT lymphoma involving the lungs and the stomach with classic histology, radiological features, and clinical behaviour.

\section{Author contributions}

All authors had full access to the data, contributed to the study, approved the final version for publication, and take responsibility for its accuracy and integrity.

Concept or design: All authors.

Acquistition of data: CPM Lam.

Analysis or interpretation of data: CPM Lam.

Drafting of the manuscript: CPM Lam.

Critical revision for important intellectual content: All authors.

\section{Conflicts of interest}

The authors have disclosed no conflict of interest.

\section{Funding/support}

This research received no specific grant from any funding agency in the public, commercial, or not-for-profit sectors. 


\section{Ethics approval}

This study was conducted in accordance with the principles outlined in the Declaration of Helsinki. The patient provided verbal informed consent.

\section{References}

1. Bacon CM, Du MQ, Dogan A. Mucosa-associated lymphoid tissue (MALT) lymphoma: a practical guide for pathologists. J Clin Pathol 2007;60:361-72.

2. Zhang WD, Guan YB, Li CX, Huang XB, Zhang FJ. Pulmonary mucosa-associated lymphoid tissue lymphoma: computed tomography and $18 \mathrm{~F}$ fluorodeoxyglucose- positron emission tomography/computed imaging findings and follow-up. J Comput Assist Tomogr 2011;35:608-13.

3. Yoshino T, Ichimura K, Mannami T, et al. Multiple organ mucosa-associated lymphoid tissue lymphomas often involve the intestine. Cancer 2001;91:346-53.

4. de Boer JP, Hiddink RF, Raderer M, et al. Dissemination patterns in non-gastric MALT lymphoma. Haematologica 2008;93:201-6.

5. Konoplev S, Lin P, Qiu X, Medeiros LJ, Yin CC. Clonal relationship of extranodal marginal zone lymphomas of mucosa-associated lymphoid tissue involving different sites. Am J Clin Pathol 2010;134:112-8. 